Musées, Patrimoine et Culture scientifiques et techniques

$168 \mid 2016$

novembre-décembre 2016

\title{
Santé et sécurité au travail dans le secteur de la conservation du patrimoine à travers l'expérience du programme Joconda
}

\section{Virginie Serrière}

\section{(2) OpenEdition}

12 Journals

\section{Édition électronique}

URL : http://journals.openedition.org/ocim/1718

DOI : 10.4000/ocim. 1718

ISSN : 2108-646X

Éditeur

OCIM

\section{Édition imprimée}

Date de publication : 1 novembre 2016

Pagination : 25-30

ISSN : 0994-1908

\section{Référence électronique}

Virginie Serrière, «Santé et sécurité au travail dans le secteur de la conservation du patrimoine à

travers l'expérience du programme Joconda », La Lettre de I'OCIM [En ligne], 168 | 2016, mis en ligne le 01 novembre 2017, consulté le 10 décembre 2020. URL : http://journals.openedition.org/ocim/1718 DOI : https://doi.org/10.4000/ocim.1718

Ce document a été généré automatiquement le 10 décembre 2020.

Tous droits réservés 


\title{
Santé et sécurité au travail dans le secteur de la conservation du patrimoine à travers l'expérience du programme Joconda
}

\author{
Virginie Serrière
}

Les acteurs de la santé et de la sécurité au travail

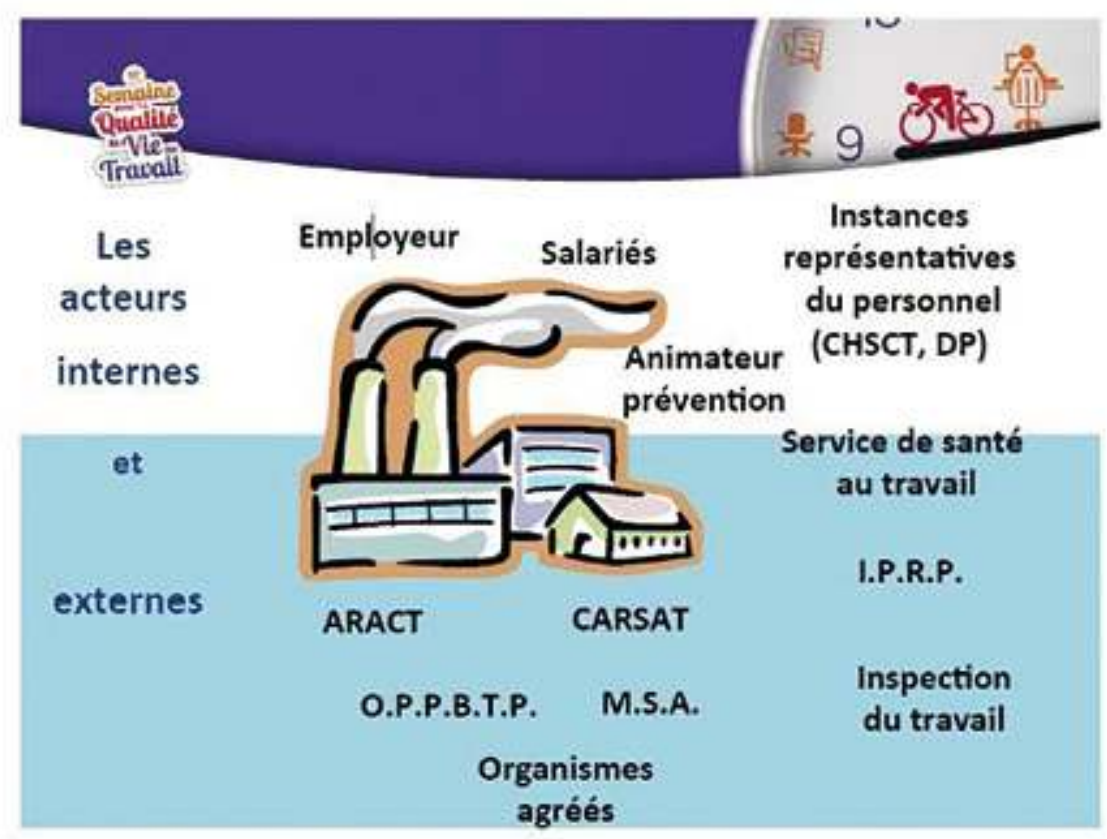

(c) DR

La Carsat Sud-Est, Caisse d'Assurance Retraite et de Santé au Travail, a participé au Programme Européen Joconda dans le but d'accompagner les professionnels de la 
conservation-restauration du patrimoine dans leur démarche d'intégration de la méthodologie institutionnelle de prévention des risques professionnels.

L'objectif de cette participation était d'insuffler le plus en amont possible les messages et bonnes pratiques de prévention à une profession prenant conscience des risques qu'elle encourt au quotidien dans le cadre de ses différentes activités. Leur souhait : fédérer l'ensemble des disciplines associées à ce secteur pour organiser et structurer une démarche générale de prévention des Accidents du Travail et de survenue de Maladies Professionnelles donnant l'opportunité d'une coopération européenne pluridisciplinaire innovante en matière de santé et sécurité au travail.

\section{La Direction des Risques Professionnels à la Carsat}

3 Organismes de la Sécurité Sociale, les Carsat sont les référents institutionnels régionaux de gestion des retraites mais aussi de prévention des risques professionnels. Leur champ d'action, dans ce domaine, concerne les entreprises et les salariés du régime général.

Dans ce contexte réglementaire, la Carsat a pour mission de calculer, fixer et notifier chaque année aux entreprises leur taux de cotisations lié aux accidents du travail et aux maladies professionnelles AT/MP survenus. Ce taux permet de calculer la contribution de chaque entreprise aux dépenses résultant des AT/MP. Ces cotisations payées par l'entreprise assurent les salariés contre les risques d'accident du travail, de trajet ou de maladie professionnelle. La Carsat se définit comme l'assureur social des entreprises du régime général.

5 En région, la Carsat Sud-Est agit prioritairement auprès d'entreprises et/ou de secteurs à forte sinistralité ou exposés à des risques importants en matière d'AT/MP. Ces priorités d'action sont déterminées par les programmes fixés au plan national par la Caisse Nationale d'Assurance Maladie des Travailleurs Salariés, organisme de tutelle paritaire, en tenant compte des évolutions socio-économiques des territoires et des avis énoncés par ses partenaires sociaux.

6 Pour cela, elle dispose d'un éventail d'actions pour aider les employeurs à prévenir les risques et protéger les salariés : informations, conseils, formations, interventions de préventeur en entreprises, incitations financières, contrôles, laboratoire de chimie et aide à la conception...

7 Des programmes nationaux et régionaux sont édictés sur des risques prioritaires (troubles musculo-squelettiques, chute de Hauteur dans le BTP, agents cancérogènes mutagènes et reprotoxiques...), et dans des secteurs d'activité ciblés (EHPAD, bricolage, restauration collective...).

8 À ces programmes viennent s'ajouter des actions basées sur la coopération avec d'autres acteurs institutionnels de la prévention des risques professionnels et des partenaires locaux : services de santé au travail ; directions régionales des entreprises, de la concurrence, de la consommation, du travail et de l'emploi ; associations régionales de l'amélioration des conditions de travail ; fédérations professionnelles....

9 Les interlocuteurs directs en entreprise sont représentés par les contrôleurs de sécurité et ingénieurs conseils repartis sur des secteurs géographiques ou rattachés aux services d'assistance spécifiques. 
10 Leurs interventions sont directement financées par les cotisations patronales et peuvent être déclenchées par une demande directe des entreprises, par les contrôleurs de la CARSAT, les inspections du travail et les services de santé au travail.

11 Dans le secteur des métiers de la conservation et restauration des collections du patrimoine, la Carsat Sud-Est a mené depuis 2007 de nombreuses actions, en partenariat avec le Laboratoire de Conservation, Restauration et Recherche à Draguignan (LC2R).

$12 \quad 2007$

13 - sollicitation de Jacques Rebière du Centre Archéologique du Var conjointement avec le docteur Michel suite à des lymphopénies identifiées au sein des équipes de Draguignan afin d'identifier l'origine de ces pathologies et d'effectuer des prélèvements de composés organiques volatils au sein des ateliers;

14 - désarmement du générateur RX et tirs radio effectués par une entreprise spécialisée compétente, mais aussi... conseils apportés sur la mise en œuvre et le stockage des produits chimiques incompatibles ;

15 - découverte de formaldéhyde dans les résultats des prélèvements de composés organiques volatils effectués.

$16 \quad 2008$

17 - analyses complémentaires pour identifier l'origine et les sources d'émission de formaldéhyde dans les ateliers alors que le produit est absent au sein du LC2R ;

18 - identification de sources de pollution naturelles au formaldéhyde liées à la présence de microflore bactérienne sur des lingots de fer oxydés provenant de fouilles de fonds marins.

$19 \quad 2009$

20 - accompagnement du LC2R pour gérer les risques de pollutions des collections de plomb à l'aide de prélèvements surfaciques réalisés avec des lingettes spécifiques ;

21 - mise en évidence de l'importance du processus de nettoyage des vêtements de travail et gants souillés, sources de pollution des zones saines et des accessoires administratifs.

$22 \quad 2010$

23 - colloque à Draguignan : présentation des résultats "Apport de la métrologie dans l'évaluation des expositions au risque chimique"

24 2011-2013

25 - participation au projet Européen Joconda

$26 \quad 2014$

27 - prélèvements atmosphériques des émissions générées lors du traitement de surface laser de sarcophages en plomb ;

28 - conclusion sur les techniques alternatives au traitement laser des collections métalliques ;

29 - colloque à Figanières : présentation et conception des équipements et lieux de travail destinés aux métiers de la conservation/restauration \& Prévenir les risques liés à la soustraitance : Qui fait quoi en santé \& sécurité?

30 D'autres actions complémentaires sont par ailleurs menées pour étayer l'évaluation des expositions professionnelles de ce secteur d'activité : 
31 - muséum d'Histoire naturelle d'Aix-en-Provence : travail autour de la problématique des herbiers traités à l'arsenic et au mercure, des collections animalières conservées dans des bocaux de formaldéhyde ;

32 - conception du Laboratoire Archéologique d'Aix-en-Provence avec une intégration le plus en amont possible des principes généraux de prévention et bonnes pratiques dans les projets de construction ;

33 - musée départemental Arles Antique : conseils en matière de prévention du risque chimique concernant la gestion et l'utilisation des produits, ainsi que sur le captage des poussières issues du micro-sablage ;

34 - INRAP (Institut national de recherches archéologiques préventives) : prélèvements atmosphériques de polluants émis lors des fouilles effectuées sur sites et sols pollués.

\section{Participation de la Carsat Sud-Est au projet européen Joconda}

\section{La réglementation française en matière de prévention des Risques Professionnels} hiérarchie des principes généraux de prévention, dès lors que les risques n'ont pu être évités ;

37 - la loi 91-1414 du 31 décembre 1991 qui pose l'obligation pour l'employeur d'assurer la sécurité et la santé physique et mentale des travailleurs, met en œuvre des principes généraux de prévention des risques professionnels et prévoit l'obligation de procéder à l'évaluation des risques ;

38 - le Décret d'application 2001-1016 du 5 novembre 2001 qui porte obligation pour l'employeur de créer et de conserver un document comportant les résultats de l'évaluation des risques (document unique), de le mettre à jour annuellement ou lors de toute modification importante et de le tenir à la disposition des représentants des salariés.

39 L'objectif recherché dans la participation au projet Joconda consistait à accompagner le transfert d'une réflexion basée sur la protection des travailleurs vers une approche règlementaire basée sur la préservation de l'atteinte physique et psychique des travailleurs en appliquant la méthodologie institutionnelle basée sur :

40 - la mise en œuvre d'une démarche de prévention primaire consistant à éliminer ou réduire à la source les différents facteurs de risques présents dans l'organisation du travail (contrairement aux démarches de prévention secondaire et tertiaire basées sur les caractéristiques personnelles et les mécanismes des individus en les outillant individuellement contre les différents risques identifiés) ;

41 - le respect des 9 principes généraux de prévention :

42 . éviter les risques, en supprimant le danger ou l'exposition au danger ; 
43 . évaluer les risques, en appréciant l'exposition au danger et l'importance du risque afin de prioriser les actions de prévention à mener ;

44 . combattre les risques à la source, en intégrant la prévention le plus en amont possible, notamment dès la conception des lieux de travail, des équipements ou des modes opératoires ;

45 . adapter le travail à l'Homme, en tenant compte des différences interindividuelles, dans le but de réduire les effets du travail sur la santé ;

46 . tenir compte de l'évolution de la technique, en adaptant la prévention aux évolutions techniques et organisationnelles;

47 . remplacer ce qui est dangereux par ce qui l'est moins, en évitant l'utilisation de procédés ou de produits dangereux lorsqu'un même résultat peut être obtenu avec une méthode présentant des dangers moindres ;

48 . planifier la prévention en intégrant technique, organisation et conditions de travail, relations sociales et environnement ;

49 . donner la priorité aux mesures de protection collective et n'utiliser les équipements de protection individuelle qu'en complément des protections collectives si elles se révèlent insuffisantes ;

50 . donner les instructions appropriées aux salariés, en formant et informant les salariés afin qu'ils connaissent les risques et les mesures de prévention.

\section{La démarche de prévention des risques professionnels}

51 Une démarche de prévention des risques professionnels se construit en impliquant tous les acteurs concernés et en tenant compte des spécificités de l'entreprise. Elle doit être guidée par trois valeurs essentielles.

\section{La personne}

52 L'employeur, l'encadrement et les salariés sont impliqués dans la démarche de prévention des risques professionnels. Les méthodes de management utilisées doivent être compatibles avec une éthique du changement qui respecte la personne.

\section{La transparence}

53 La maîtrise des risques implique pour l'employeur et l'encadrement :

54 - l'affichage des objectifs visés (en matière d'amélioration de la santé, de la sécurité et des conditions de travail);

55 - l'engagement et l'exemplarité du chef d'entreprise et de l'encadrement dans la mise en œuvre de la démarche de prévention (implication personnelle et mise à disposition des moyens nécessaires);

56 - la prise en compte de la réalité des situations de travail ;

57 - la clarté dans la communication sur la santé et la sécurité au travail. 


\section{Le dialogue social}

58 L'adhésion du personnel est une condition indispensable dans la mise en place d'une politique de prévention des risques. Cela implique d'associer les salariés et les instances représentatives du personnel (CHSCT et délégués du personnel) à la mise en œuvre de celle-ci.

\section{L'accompagnement spécifique de la profession dans l'analyse d'un accident du travail}

Comment accompagner le secteur d'activité lors de la réalisation de la première étape de l'évaluation des risques, à savoir l'identification des dangers et situations de travail dangereuses ? La plus-value et la participation de la Carsat dans le projet Joconda se sont centrées sur cette problématique comme le rappelle l'extrait suivant du rapport final Joconda :

Le programme adopté sous le nom de Joconda constitue une démarche naissante de prise de conscience, par une profession encore jeune, des risques qu'elle encourt dans le cadre de sa propre activité.

61 À l'origine de la réunion de Draguignan, coorganisateur des deux colloques consacrés à ce thème en 2007 et 2010, le LC2R Draguignan (CAV Toulon) a mis son réseau au service de cette démarche. Ainsi, des professionnels des domaines de la conservation-restauration, de l'enseignement supérieur, des associations de conservateurs-restaurateurs, de la santé/sécurité au travail, des ergonomes, des responsables de structures de restauration... ont été regroupés dans ce qui constitue le groupe national le plus important, numériquement, de Joconda.

L'opportunité d'une coopération européenne associant à ce groupe prédéfini des professionnels de la communication, des chercheurs européens sur des programmes ambitieux est une garantie de prise en considération du problème abordé par Joconda, suivant une multiplicité d'approches.

Pour autant, il est très vite apparu un décalage entre les réflexions des professionnels en santél sécurité et la pratique (ou le niveau de prise de conscience) des praticiens de la conservationrestauration. Les conservateurs-restaurateurs n'exercent pas dans les mêmes conditions statutaires, ce qui nuit à une diffusion des bonnes pratiques (beaucoup exercent seuls dans des structures "artisanales" ou uninominales et de fait se trouvent isolés).

64 Il est également apparu prioritaire aux conservateurs-restaurateurs de mesurer le niveau de risque encouru et d'observer le niveau de prise de conscience de ces risques : les interventions menées par les conservateurs-restaurateurs sur les objets archéologiques terrestres et sous-marins, artistiques, ethnographiques, historiques, industriels sont de plusieurs ordres : mécanique, chimique et/ou électrochimique.

Ces différentes opérations nécessitent l'emploi d'outils, de machines, de produits chimiques qui s'accompagnent de risques souvent méconnus, mésestimés voire dangereux pour le long terme. Selon la médecine du travail, cette profession est considérée comme un métier à hauts risques.

Le projet Joconda a regroupé la Belgique, l'Espagne, la France, la Hongrie, l'Italie, la Pologne, la Slovénie et la Suisse. Le LC2R Draguignan a été désigné chef de file du groupe français. L'ensemble des rapports scientifiques (Rapport intermédiaire juin 2012, rapport final septembre 2013 et saisie en ligne $d u$ rapport final partenariats Leonardo 2011 (Rapport Final E-FORMS) sur la base européenne EST (European Shared Treasure)) a été rédigé par Françoise Mielcarek. 

conservation-restauration du patrimoine. Améliorer la santé et la sécurité sur le lieu de travail est une initiative novatrice dans ce domaine. En effet, la santé/sécurité au travail en conservationrestauration du patrimoine a longtemps été un sujet "tabou" ou tout simplement non pris en compte par ces professionnels et les institutions. La grande majorité d'entre eux ne disposent pas ou peu de formation scientifique. La notion de risques ne fait pas partie de leur culture ni de leur réflexion. En outre, il existe peu de formations qui intègrent l'enseignement de la sécurité, des bonnes pratiques de prévention spécifiquement adaptées à la conservation-restauration du patrimoine. Un autre élément est à prendre en considération, la sécurité engendre des investissements lourds difficilement supportables pour la majorité des structures d'où la tentation de sous-estimer voire nier les situations dangereuses. Il s'agit donc de sensibiliser et d'insuffler une prise de conscience des risques engendrés et subis par ces professionnels sans oublier l'impact sur l'environnement.

Suite à la première réunion, divers inventaires des risques ont été réalisés par les partenaires espagnols, français et polonais. Ces documents ont rapidement démontré des approches différentes, ce qui a poussé les partenaires français à organiser à Marseille au siège de la Carsat Sud-Est, une réunion avec des professionnels de la santé/sécurité au travail. Son but principal était de préciser le vocabulaire et les démarches à suivre. En effet, la priorité était de définir en amont ce que l'on entendait par la notion "d'identification des risques" et la manière dont on définit les notions de risque, de danger, d'accident de travail, de maladie professionnelle... Les documents fournis par nos partenaires étaient certes détaillés mais trop généralistes. Ces inventaires parfaitement appropriés et adaptés à des centres de recherche, et à des laboratoires scientifiques n'illustraient pas l'ensemble des risques spécifiques auxquels les conservateurs-restaurateurs étaient confrontés quotidiennement. En effet, en raison de leurs différents statuts (indépendants, autoentrepreneurs, salariés, fonctionnaires), et de leurs spécialités, les risques et les sources de danger sont multiples et nécessitent une enquête approfondie afin de fournir un document crédible et pertinent.

Il a donc été décidé de mettre au point un questionnaire destiné aux conservateurs/restaurateurs du patrimoine. Il doit être utilisé comme une aide au repérage des situations dangereuses. Il comprend 10 questions portant sur l'activité professionnelle (spécialité), la fonction, le travail à temps complet / à temps partiel, le statut (indépendant, salarié, autoentrepreneur ou fonctionnaire), le cadre de travail (en équipe ou isolé), le niveau de protection mis à disposition (individuelle, collective ou absence de protection), l'existence ou non d'un suivi médical, le sexe, le nombre d'années d'expérience, le nombre de salariés exposés.

Le tableau identifie les risques repérés et l'environnement de travail (atelier/laboratoire - lieu d'accueil collectif - en extérieur, sur chantier). Les risques inventoriés sont au nombre de 26 rubriques. Cinq questions complémentaires portent sur le nombre d'accidents déclarés par an, les types d'accidents déclarés, les secteurs ou activités touchés et l'analyse des accidents de travail. Trois questions supplémentaires sont en outre posées: "ce qui dérange le plus", "ce qui peut faire mal" et "conseils prioritaires à donner aux nouveaux venus".

\section{Pour conclure}

71 La participation de la Carsat aux différentes réunions du projet Joconda a permis d'accompagner la profession sur cette première étape primordiale et nécessaire pour mener à bien l'Évaluation des Risques Professionnels.

La Lettre de l'OCIM, 168 | 2016 
72 Il s'agissait de mettre en évidence les conditions d'exposition, en analysant le travail réel des opérateurs dans le cadre de la recherche de situations de travail dangereuses (voir le Rapport final Joconda : première étape d'identification des risques).

73 La démarche retenue pour l'ensemble des disciplines du secteur d'activité a permis également de concrétiser ces situations de travail au moyen :

74 - d'un découpage spécifique selon 3 activités distinctes, et ce, quel que soit la spécificité (voir le Rapport final Joconda : Laboratoire/Musées/Travaux en extérieur) ;

75 - du croisement entre activité propre et prestations mandatées par un Donneur d'Ordre (voir les articles du colloque Figanières : Entreprise Utilisatrice/Entreprise Extérieure et Plans de Prévention).

76 Cette méthodologie a été un moyen de parvenir pour la profession à l'exhaustivité de tous les Risques Professionnels pour tous les métiers concernés. Ainsi, le rapport final du projet JOCONDA précise dans sa conclusion :

77 Ces approches sont certes différentes mais complémentaires, chaque partenaire, en fonction de sa spécialité apporte son savoir-faire et son expérience. Tout l'intérêt et la complexité de ce projet Joconda est de faire travailler ensemble des partenaires européens qui disposent chacun d'une formation, d'une règlementation, d'un cadre professionnel spécifique.

78 Préalablement à toute étude sur les risques, nous avons donc voulu estimer le niveau de conscience des risques et des situations dangereuses des acteurs concernés. C'est pourquoi, un "prototype" d'enquête a été élaboré et fut testé auprès de conservateurs-restaurateurs de diverses spécialités et de statuts variés. Le questionnaire vise à proposer une méthodologie et des concepts jusqu'alors méconnus des conservateurs-restaurateurs tel que la notion "d'arbre des causes" afin de faciliter une meilleure prise en compte des facteurs d'accidents et cela le plus en amont possible. La dissémination des résultats de ce travail contribuera à proposer cette enquête dans d'autres pays et aboutira à un "état de l'art" plus complet.

79 Par ailleurs, il est nécessaire de mieux faire comprendre les enjeux des risques professionnels en conservation-restauration du patrimoine au niveau des commanditaires par la rédaction d'un livre blanc. Il a été rappelé que les conditions de travail des prestataires sont le plus souvent ignorées voire négligées face à l'exigence des résultats et de garantie de conservation des cuvres.

80 Le projet Joconda vise donc à déterminer les risques encourus par les conservateurs/restaurateurs au sein des laboratoires/ateliers, centres d'accueil collectif et sur site, en extérieur. Les principaux axes du projet consistent à :

81 - identifier et catégoriser les risques professionnels encourus par les conservateurs/restaurateurs,

82 - mettre en place les "bonnes pratiques" afin d'informer et de motiver les conservateurs/ restaurateurs et les conservateurs à appliquer les règles de sécurité et santé au travail,

83 - définir les lignes directrices de formation en santé et sécurité au travail adaptées aux conservateurs-restaurateurs du patrimoine comprenant les connaissances, aptitudes et compétences, évaluées par le biais du CEC (Cadre européen des certifications) et conformément à la reconnaissance des acquis de la mobilité (ECVET),

84 - établir un premier bilan de la situation en Europe (approches, lois et formations nationales) afin de créer une formation européenne en santé et sécurité au travail spécifiquement adaptée à la profession de conservateurs-restaurateurs du patrimoine et conforme aux dispositions du système européen d'éducation et formation professionnelle selon le cadre de compétences des qualifications européennes en utilisant des supports multimédias, 
- créer un réseau européen,

- élaborer un "livre blanc" adressé aux institutions responsables des marchés publics afin de les sensibiliser aux conditions de travail des conservateurs-restaurateurs et de leur permettre de prendre en considération les règles de sécurité et de santé au travail.

\section{Exemple d'identification de situation de travail dangereuse}

Dans cet exemple de situation de travail, comment aborder l'identification des risques, comment identifier les dangers, comment énoncer la situation de travail dangereuse en employant la même sémantique?
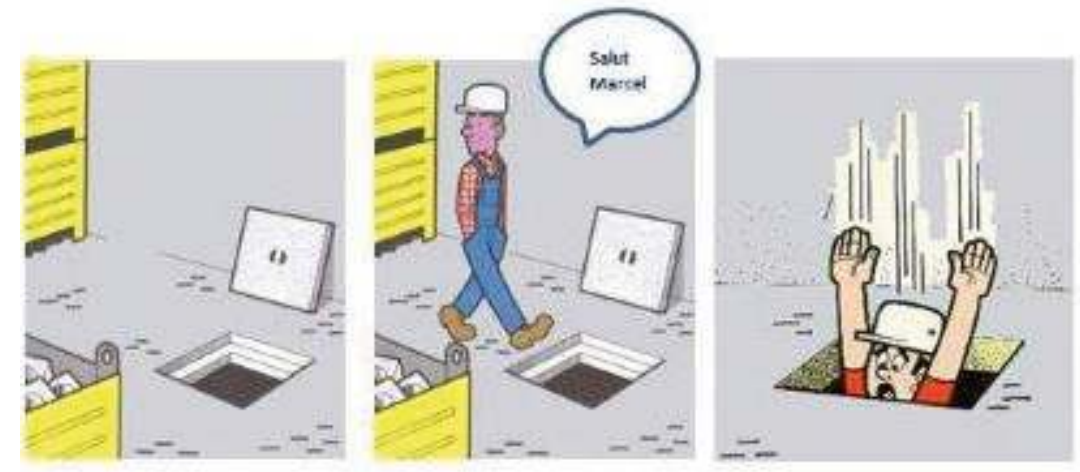

\section{Présentation des définitions consensuelles institutionnelles :}

Dommage : lésion physique ou atteinte à la santé

Danger : source potentielle de dommage, ce qui fait mal

Risque : probabilité d'apparition d'une lésion ou atteinte à la santé

Situation dangereuse : environnement de travail dans lequel une personne est exposée à un ou plusieurs dangers

Événement déclenchant : facteur susceptible de causer un dommage

Accident : évènement imprévu générant lésion ou atteinte à la santé 


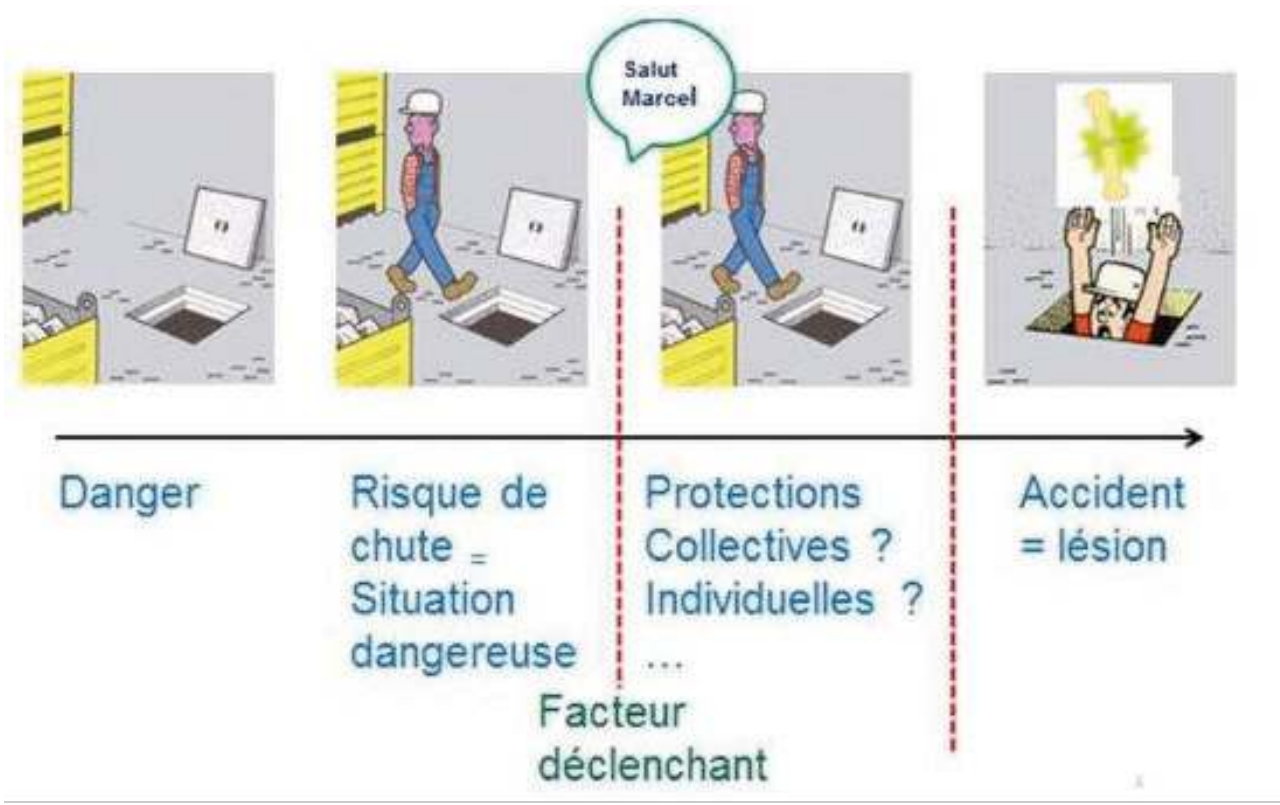
évoluant au cours de sa journée à proximité d'une trémie non protégée (absence de
protection collective périphérique ou de platelage sécurisé), disposant de chaussures de
sécurité et d'un casque de chantier

Dommage : fracture des deux fémurs

\section{RÉSUMÉS}

Partant du constat des risques professionnels encourus dans le domaine de la conservation/ restauration du patrimoine et de la nécessité de mener des actions de prévention, le projet européen Joconda consiste notamment à identifier et catégoriser ces risques, mettre en place les bonnes pratiques de sécurité et santé au travail et définir dans ce domaine les lignes directrices de formation adaptées.

\section{INDEX}

Mots-clés : santé, sécurité, prévention 


\section{AUTEUR}

\section{VIRGINIE SERRIÈRE}

contrôleur Sécurité à la Carsat Sud-Est

virginie.serriere@carsat-sudest.fr 